\title{
Energy considerations in accelerating rapid shear granular flows
}

\author{
S. P. Pudasaini and B. Domnik \\ University of Bonn, Steinmann Institute, Department of Geodynamics \& Geophysics, Nussallee 8, 53115 Bonn, Germany
}

Received: 18 June 2008 - Revised: 7 May 2009 - Accepted: 7 May 2009 - Published: 20 May 2009

\begin{abstract}
We present a complete expression for the total energy associated with a rapid frictional granular shear flow down an inclined surface. This expression reduces to the often used energy for a non-accelerating flow of an isotropic, ideal fluid in a horizontal channel, or to the energy for a vertically falling mass. We utilize thickness-averaged mass and momentum conservation laws written in a slope-defined coordinate system. Both the enhanced gravity and friction are taken into account in addition to the bulk motion and deformation. The total energy of the flow at a given spatial position and time is defined as the sum of four energy components: the kinetic energy, gravity, pressure and the friction energy. Total energy is conserved for stationary flow, but for non-stationary flow the non-conservative force induced by the free-surface gradient means that energy is not conserved. Simulations and experimental results are used to sketch the total energy of non-stationary flows. Comparison between the total energy and the sum of the kinetic and pressure energy shows that the contribution due to gravity acceleration and frictional resistance can be of the same order of magnitude, and that the geometric deformation plays an important role in the total energy budget of the cascading mass. Relative importance of the different constituents in the total energy expression is explored. We also introduce an extended Froude number that takes into account the apparent potential energy induced by gravity and pressure.
\end{abstract}

\section{Introduction}

There is a wide spectrum of applications for granular flow, ranging from large-scale snow, rock or debris avalanches in nature, to small scale transport of granular materials in industrial handling and production processes. The energy associ-

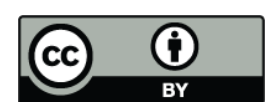

Correspondence to: B. Domnik (domnik@geo.uni-bonn.de) ated with such flows is a major concern to avalanche dynamicists with respect to avalanche defense, hazard mitigation and planning. Estimation of the total energy carried out by the avalanching mass is important for civil engineers in designing structures and spacing because they must withstand at least the total destructive power of the avalanche. As an example, cone-shaped earthen mounds are frequently built in the lower part of the track and the run-out zone to retard and dissipate the destructive power of an avalanche by absorbing its energy from reducing velocity and shortening its flow path. Thus, the avalanche stops before it reaches the area needing protection (Rao, 1985; Pudasaini and Hutter, 2007). Simple energy balance is often used to determine the runout efficiency of the events. To analyze basic features, like the horizontal displacement $L$ and the vertical height drop $H$, the energy conservation in the rigid mass model (Heim, 1932) is still widely used (Hsü, 1975; Ui, 1983; Erismann and Abele, 2001). In this simple model a single block with mass $m$ steadily slides down an incline with uniform basal friction. A comparison of the energy at the beginning and end of the motion leads to the equation $g m H=W$, with the gravitation constant $g$ and the work $W$ done by friction. By assuming Coulomb friction, $F=\mu m g$, where $\mu$ is the effective coefficient of friction, one obtains $H / L=\mu$, whose reciprocal is a measure of the efficiency of mass movement (Ward and Day, 2006). This simple expression can be used to get information about flow characteristics without requiring the full equations of motion or determining the friction parameters from the experimental or field observation.

Recently, the energy mechanics of geophysical mass flows has attracted interest. Gwiazda (2005) considered kinetic and pressure energies for avalanche flows. Similarly, Dutykh and Dias (2009), Castro et al. (2006), and Fine et al. (2003) addressed the kinetic and gravitational energy for tsunami waves. Jin and Wen (2004) and Noelle et al. (2006, 2007) took into account the kinetic, gravitational and pressure energies for steady state shallow water flow in Cartesian coordinates with a lake at-rest conditions. However, none of these

Published by Copernicus Publications on behalf of the European Geosciences Union and the American Geophysical Union. 
papers involves the role of the dissipative frictional resistance, and thus the entire energy of the flow was not considered. Bartelt et al. $(2005,2006)$ and Buser and Bartelt (2009) studied the energetics of snow avalanches by showing how frictional processes taking place at the basal surface are related to dissipative mechanisms within the avalanching body. They wrote an energy balance for thickness averaged flows, including the production of kinetic energy associated with the random movement of the snow granules; however, they always assumed a constant flow height and steady-state flow. Thus, they were not able to deduce how changes in flow height affected the overall energy balance and therefore the production of random energy.

In this paper, we focus on the general aspects of deformable avalanching mass rapidly sliding down a slope and the associated total energy budget carried out by the flow. We attempt to answer the question of how frictional dissipation and the gravity acceleration are linked to spatial variations in flow height and the flow velocity. To do so, we first rewrite the thickness averaged frictional granular flow equations (Savage and Hutter, 1989; Pudasaini and Hutter, 2003, 2007) in terms of a single state variable, the flow height, following Le Roux (1998). For such equations, internal deformations, which are assumed to be governed by an earth pressure coefficient, can be directly related to spatial variations of the flow height. We then present a complete total energy function for accelerating rapid granular flows that is applicable for a non-steady deformable mass sliding down inclines. Our energy expression takes into account all contributing factors, including kinetic energy, pressure potential, gravitational potential and the thermal energy due to frictional heat. The energy equation shows how variations in flow height and the net driving acceleration influence the overall energy distribution of granular masses sliding down inclines. Our analysis does not restrict itself to purely steady flows and therefore we can use the derived energy relations to constrain constitutive models for granular flows. We demonstrate that the contribution of the gravity potential and friction energies, collectively induced by the net driving acceleration, plays a crucial role in determining the total energy. In a series of simulations, we then show how and under which conditions, the term governing the internal deformations, bulk motions and the net driving acceleration of the system influence the total energy balance.

\section{Frictional granular flow equations}

We consider one-dimensional frictional granular flow equations with Coulomb basal sliding law to describe incompressible rapid shear granular flows down inclined channels (Pudasaini et al., 2008, 2005; Pudasaini and Hutter, 2007, 2003; Gray et al., 1999; Savage and Hutter, 1989). These are thickness-averaged balance laws of mass and momentum in slope-fitted coordinates in the form of non-linear hyperbolic partial differential equations:

$$
\begin{aligned}
\frac{\partial h}{\partial t}+\frac{\partial}{\partial x}(h u) & =0 \\
\frac{\partial}{\partial t}(h u)+\frac{\partial}{\partial x}\left(h u^{2}+\frac{1}{2} \beta h^{2}\right) & =g \cos \zeta(\tan \zeta-\tan \delta) h,
\end{aligned}
$$

where $h$ is the flow depth, $u$ the velocity parallel to the sliding surface, $\zeta$ the channel slope angle, $\tan \delta$ the coefficient of friction, $g$ the magnitude of gravitational acceleration, $\beta=g K \cos \zeta$ and $K=2 \sec ^{2} \phi\left(1 \mp \sqrt{1-\cos ^{2} \phi \sec ^{2} \delta}\right)-1$ is the earth pressure coefficient. This coefficient is a function of the internal $(\phi)$ and basal $(\delta)$ angles of friction that are active during extensional motion (upper sign) and passive during compressional motion (lower sign). The right-hand side of the momentum balance in Eq. (1) contains the net driving acceleration $s=g \cos \zeta(\tan \zeta-\tan \delta)$, which can be split into the acceleration due to gravity, $s_{g}=g \sin \zeta$, and the resistance due to friction, $s_{f}=-g \cos \zeta \tan \delta$. $K$ and $s$ incorporate the internal interaction of the media with itself and its interaction with the basal surface. Note that $s$ is the source term, which makes the system of equations inhomogeneous. If the gravity is exactly balanced by friction, the mass is only subject to internal deformation as modeled by the term $\partial\left(0.5 \beta h^{2}\right) / \partial x$, which corresponds to the pressure gradient induced by the free-surface and the anisotropy (normal stress effect) in the granular material.

\section{Rapid shear flows}

The flows of granular material down a silo gate, or in the form of dam-break flows, are essentially rarefied flows $(\mathrm{Pu}-$ dasaini et al., 2005, 2007; Mangeney et al., 2000). One particularly interesting case is the granular flow down a steep rectangular chute, where the material is uniformly fed from the silo gate (Pudasaini and Kröner, 2008). In such a situation the velocity can be expressed locally as a function of the flow depth, i.e., $u=u(h)$. This simplifies the situation and reduces the number of field variables from two to one, namely, the flow depth. With this, Eq. (1) take the form $\left(u^{\prime}=d u / d h\right)$ :

$$
\left(\begin{array}{cc}
1 & h u^{\prime}+u \\
h u^{\prime}+u & 2 h u^{\prime} u+u^{2}+\beta h
\end{array}\right)\left(\begin{array}{l}
\partial h / \partial t \\
\partial h / \partial x
\end{array}\right)=\left(\begin{array}{l}
0 \\
s h
\end{array}\right) .
$$

Since the flow is driven by the net driving acceleration, the entire analysis depends on the parameter $s$. Depending on whether it is zero, positive or negative, the flow as a whole would be non-accelerating, accelerating or decelerating, respectively. However, in each case the individual particles may accelerate or decelerate due to the free surface gradient. Here, the classical (original) shallow water or SaintVenant (1871) equations differ from our equations (Bouchut et al., 2003; Rudenko et al., 2007). In the shallow water model, the momentum transfer is only due to the free-surface 
(hydraulic) gradient of the flow. The model Eq. (1) reduce to the shallow water equations if $K=1$ and $\zeta=\phi=\delta=0$, which implies that the net driving force vanishes.

\section{Total energy in rapid granular flows}

Le Roux (1998) derived an energy function for nonhomogeneous shallow-water equations which were written in Cartesian (horizontal-vertical) coordinates. The nonhomogeneity emerges from the bottom elevation as measured from a horizontal datum. A ramp between two horizontal steps with two singularities is defined, on which rarefaction (thinning of the medium depth) of the flow takes place. No basal friction is considered. Therefore, the flow is driven by the free-surface (pressure) gradient and the gradient of the bottom elevation, which is treated as the source term. Although Le Roux did not derive the total energy for this situation (he neglected the potential energy due to gravity and the internal energy induced by friction) this concept is still useful. If the flow takes place in a steep slope, simulations produce better results when coordinates are defined by the slope instead of Cartesian coordinates. Therefore, we defined the coordinates along the slope and normal to it. As a consequence we do not need to consider the bottom elevation function that was treated before as the source term by Le Roux. Instead, the source term is composed of the gravity component along the channel minus the Coulomb friction, which has not yet been considered. In the present analysis, rarefaction can take place down the entire plane. We closely follow Le Roux (1998).

\subsection{Introduction of the energy function}

\section{Absence of the net driving force}

We start our analysis of a system when $s=0$. Since $h \neq 0$, the matrix in Eq. (2) is necessarily singular, so the corresponding determinant must vanish, implying:

$h\left(u^{\prime}\right)^{2}-\beta=0$.

This is an exact ordinary differential equation, which can be solved to yield $u \pm 2 \sqrt{\beta h}=\Lambda$, where $\Lambda$ are constants of integration. These are Riemann invariants and remain constant along the flow characteristics $d x / d t=u \pm c$, where $c=\sqrt{\beta h}$ is the wave celerity. There is some experimental evidence that Eq. (3) has some validity. By applying geometric arguments, Bartelt et al. (2007) also derived a similar relationship between $h$ and $u$, more precisely, $h \propto u^{2}$. They compared this formula to actual granular flows and showed how the tails which are near steady state - agree with this result.

\section{Presence of the net driving force}

One immediate observation is that Eq. (3) no longer holds if $s \neq 0$. Therefore, the usual Riemann invariants do not play a role (Le Roux, 1998). We introduce a function $\mathcal{E}$ to analyze the system for the general case:

$\mathcal{E}^{\prime}(h)=\beta-h\left(u^{\prime}\right)^{2}$.

Below, we will show that $\mathcal{E}$ is an energy function, which is related to the total energy of the system. This is the first energy function that we consider. By combining Eq. (4) with Eq. (2) we obtain:

$\mathcal{E}^{\prime}(h) \frac{\partial h}{\partial x}=s, \quad \frac{\partial \mathcal{E}(h)}{\partial x}=s$.

This shows that the spatial derivative of $\mathcal{E}$ is the net acceleration of the system. Again from the mass balance in Eq. (2) and applying the chain-rule of differentiation, we get:

$\frac{\partial \mathcal{E}(h)}{\partial t}=-s \frac{d}{d h}(h u)$.

The time rate of $\mathcal{E}$ is also related to the net driving acceleration, and therefore the energy function $\mathcal{E}$ is constant if the system does not accelerate. Even if the flow is stationary (which here means the flux is constant with respect to the flow depth), $\mathcal{E}$ is not constant (although the time rate of $\mathcal{E}$ vanishes) because this does not apply to the partial derivative with respect to space. Equations (5) and (6) lead to the following representation of the energy function

$\mathcal{E}(h)=s_{g}\left(x-x_{d}\right)+s_{f} x+\lambda(t)$,

where $x_{d}$ is the constant of integration, which is the distance from the point of the mass release along the channel to the point where the flow hits the horizontal reference datum. Gravitational potential energy is maximum at the position of the mass release where the frictional dissipation is minimum (or zero), and vice versa at the reference datum. This is the reason for choosing different but appropriate references for $s_{g}$ and $s_{f}$, respectively, in Eq. (7). Since the "granulargraph" varies in space for rarefied granular flows on inclines, $\partial h / \partial x \neq 0$. With $\mathcal{E}_{x t}=\mathcal{E}_{t x}$, this leads to the following exact representation of the granular-flux or momentum,

$h u=\eta h+\mu$,

where $\eta$ and $\mu$ are constants of integration and $\eta$ is the flux gradient, so has a dimension of a velocity. This establishes a simple and explicit linear relationship between the flux and the flow height.

An alternative representation of $\mathcal{E}$ is achieved by combining Eq. (4) with Eq. (8):

$\mathcal{E}(h)=\frac{1}{2}(u-\eta)^{2}+\beta h$.

Combining the two equivalent representations in Eqs. (7) and (9), we obtain

$$
\begin{aligned}
\mathcal{E}(h) & =\frac{1}{2}(u-\eta)^{2}+\beta h \\
& \equiv s_{g}\left[\left(x-x_{d}\right)-\eta t\right]+s_{f}[x-\eta t]+\lambda_{0},
\end{aligned}
$$


where $\lambda_{0}$ is a constant. Note that the constants $\eta, \mu$ and $\lambda_{0}$ are problem-specific and their values can be determined differently for different problems, e.g., dam-break flows, roll waves, splash flows, or a flow discharged from a silo (Le Roux, 1998; Pudasaini and Kröner, 2008). The right-hand side of Eq. (10) shows that the energy function $\mathcal{E}$ is a solitary wave, which travels with speed $\eta$. Here, $s=s_{g}+s_{f}$ is the amplifying factor of the wave and $\lambda_{0}-s_{g} x_{d}$ is the absolute shift.

\section{Riemann variables and rarefied flows}

The model Eq. (1) constitutes a system of hyperbolic partial differential equations. They can be written in the general vectorial construct of the Riemann variables $\boldsymbol{W}=\boldsymbol{W}(h, u)$ and slopes of the characteristic lines $\boldsymbol{R}_{K}=\boldsymbol{R}_{K}(h, u) \quad$ as $\partial \boldsymbol{W} / \partial t+\boldsymbol{R}_{K} \cdot \partial \boldsymbol{W} / \partial x=\boldsymbol{S}$, where $\boldsymbol{S}$ is the source term with $\boldsymbol{W}=(u+2 \sqrt{\beta h}, u-2 \sqrt{\beta h})^{T}$, $\boldsymbol{R}_{K}=(u+\sqrt{\beta h}, u-\sqrt{\beta h})$, and $\boldsymbol{S}=(s, s)^{T}$, where $T$ is the transpose. For rarefied waves, such as induced by dam-break and silo discharge, the method of characteristics and the Riemann variables can be used to construct exact solutions for unknown fields $h$ and $u$ in terms of a similarity variable $\xi=x / t$ (thus rarefaction fan). The solutions read: $(h, u)(\xi)=\left[\left(2 \sqrt{\beta h_{0}}-\xi-0.5 s t\right)^{2} / 9 \beta, 2\left(\sqrt{\beta h_{0}}+\xi-2 s t\right) / 3\right]$, where $h_{0}$ is the initial flow height.

\subsection{Constant flux and the total energy}

If there is a relatively large amount of granular material fed from the outlet of the silo or the source, and if the flow takes place in an inclined channel, the flow quickly shears down the channel and the stationary or steady-state condition (constant flux) may prevail for a long time throughout the channel (Pudasaini et al., 2007; Pudasaini and Kröner, 2008). A stationary flow can be characterized by setting $\eta=0$ in Eq. (10). Then the energy function takes the form:

$\mathcal{E}(h)=\frac{1}{2} u^{2}+\beta h \equiv s_{g}\left(x-x_{d}\right)+s_{f} x+\lambda_{0}$.

It is interesting to observe the two expressions for $\mathcal{E}$ in Eq. (11). The right-side of the expression explicitly contains $s$ but the left does not, which is influenced by $s$ intrinsically through the dynamical field quantities $u$ and $h$. Although the right-hand side does not contain $u$ and $h$ explicitly, the effect is implicit through the travel distance $x$.

In the following we consider only the left side for $\mathcal{E}$ in Eq. (11). The first term $0.5 u^{2}$ corresponds to the kinetic energy $\left(E_{\mathrm{kin}}\right)$ and the second term $\beta h$ is the pressure potential energy $\left(E_{\mathrm{pot}}^{p}\right)$ due to the pressure gradient. $\mathcal{E}$ is a convex function of $h$. The energy level goes to infinity for either $h \rightarrow \infty$ or $h \rightarrow 0$, which corresponds physically to a very large inflow height at the top of the channel (silo gate), or a very thin flowing layer at long distances in the channel, respectively. Analysis of the result is rather simple. The situation $h \rightarrow 0$ implies that,
$E_{\text {pot }}^{p}$ tends to zero but $E_{\text {kin }}$ tends to infinity. In contrast, $h \rightarrow \infty$ implies that, $E_{\text {pot }}^{p}$ tends to infinity but $E_{\text {kin }}$ reduces to zero. The energy function attains its minimum $\mathcal{E}_{\min }=0.5 \mu^{2} / h_{\min }^{2}+\beta h_{\min }$ with $h_{\min }=\mu^{2 / 3} / \beta^{1 / 3}$ for which the critical velocity is $u_{\min }=\sqrt{\beta h_{\min }}$.

We now define the total energy function $E$, which represents the total energy of the system at a given position and time (this is the second energy function that we consider):

$$
E(h)=\underbrace{\frac{1}{2} u^{2}}_{E_{\mathrm{kin}}}+\underbrace{\beta h}_{E_{\mathrm{pot}}^{p}}+\underbrace{s_{g}\left(x_{d}-x\right)}_{E_{\mathrm{pot}}^{g}}-\underbrace{s_{f} x}_{-E_{\text {fric }}},
$$

where we identify the kinetic energy as $E_{\text {kin }}$, the two potential energies by $E_{\mathrm{pot}}^{p}$ and $E_{\mathrm{pot}}^{g}$ (these are caused by the free-surface gradient of the flow with respect to the inclined sliding surface and the gravity in the direction of motion, respectively) and the thermal or internal energy induced by the frictional dissipation along the bed by $E_{\text {fric }}$. The thermal energy is distributed among the sliding mass, the basal surface and possibly to the ambient environment that collectively define a closed system. For a stationary flow, $E$ is equal to $\lambda_{0}$ and consequently a conserved quantity. If the flow is nonaccelerating, i.e., the gravity is exactly balanced by the material friction, the sum of the kinetic and the potential energy due to pressure always remains constant. This is the total mechanical energy for a flow when the net driving acceleration is neglected. The third energy function is:

$\Sigma:=E_{\mathrm{kin}}+E_{\mathrm{pot}}^{p}\left(=E-E_{\mathrm{pot}}^{g}-E_{\text {fric }}\right)$.

Otherwise, there is an additional contribution, $E_{\text {pot }}^{g}+E_{\text {fric }}$, induced by the net acceleration. This clearly demonstrates that even for a constant flux, the energy $\Sigma$ is not constant. It must incorporate the enhancing or dissipative contribution due to the non-vanishing acceleration, and take into account the gravitational and frictional forces. In general $E$ and $\Sigma$ are related by $E=\Sigma+E_{\text {pot }}^{g}+E_{\text {fric }}$. For non-accelerating and non stationary flows $E$ and $\Sigma$ differ only by $s_{g} x_{d}: E=\Sigma+s_{g} x_{d}$. For accelerating but stationary flows, the energy function $\mathcal{E}$ takes a physical meaning: $\mathcal{E}=\Sigma$.

\subsection{Extended total energy function}

For a non-stationary flow the total energy Eq. (12) is no longer a constant. Instead the following applies:

$E=\lambda_{0}-\frac{1}{2} \eta^{2}-s \eta t+u \eta$,

as deduced from Eqs. (10) and (12). The time dependence of $E$ is a consequence of the time-dependence of the flow height, which leads to the fact that the force induced by the free-surface gradient is not conservative. This becomes more clear when calculating $d E / d t$ from Eq. (14), and using the mass and momentum conservation together with Eq. (8): $d E / d t=-\eta \beta \partial h / \partial x=\beta \partial h / \partial t$, where $\eta=u+h d u / d h$ is a 
non-trivial wave speed. In the following the physical meaning of the total energy function is explained in more detail.

To get the total energy function in proper dimensional form we multiply Eq. (12) by the mass $m=\rho$ (per unit volume, where $\rho$ is the bulk density of the granular material)

$$
E(h)=\underbrace{\frac{1}{2} m u^{2}}_{E_{\mathrm{kin}}}+\underbrace{m \beta h}_{E_{\mathrm{pot}}^{p}}+\underbrace{m s_{g}\left(x_{d}-x\right)}_{E_{\mathrm{pot}}^{g}}-\underbrace{m s_{f} x}_{-E_{\mathrm{fric}}} .
$$

Note that the energies $E, E_{\mathrm{kin}}, E_{\mathrm{pot}}^{p}, E_{\mathrm{pot}}^{g}$ and $E_{\text {fric }}$ have been redefined to include the multiplicity of $m$. The contribution $E_{\text {pot }}^{g}+E_{\text {fric }}$ is of particular importance as it is driving the entire energy dynamics. The potential energy, $E_{\mathrm{pot}}^{p}+E_{\mathrm{pot}}^{g}$, attains a maximum value at the point of mass release (e.g., the silo gate) and becomes a minimum (or may even reduce to zero if the flow depth is negligible) as the flow hits the reference horizontal datum. At that moment the potential energy is transferred to the kinetic energy, $E_{\text {kin }}$, and the friction induced internal energy, $E_{\text {fric }}$.

In order to obtain the energy for an isotropic frictionless ideal fluid, we set $K=1$ and $\phi=\delta=0$. Then, with $\beta=g K \cos \zeta$ and $s_{g}=g \sin \zeta$, Eq. (15) reduces to:

$$
E(h)=\frac{1}{2} m u^{2}+m g \cos \zeta h+m g \sin \zeta\left(x_{d}-x\right) .
$$

For the energy of an ideal fluid in a horizontal channel $(\zeta=0)$ we obtain the often used form $E=0.5 m u^{2}+m g h$. Therefore, we conclude that Eq. (15) is the extended form of the total energy budget associated with rapid (or creeping) granular flows down a channel.

Finally, the most simple forms of total energies for granular flows are

$$
\begin{aligned}
& \zeta=0^{\circ}: \quad E(h)=\frac{1}{2} m u^{2}+m g K h+m g \tan \delta x, \\
& \zeta=90^{\circ}: \quad E(h)=\frac{1}{2} m u^{2}+m g\left(x_{d}-x\right) .
\end{aligned}
$$

In Eq. (17), the only deformation is due to the pressure potential and the internal anisotropic pressure $(K \neq 1)$, and resisted by the basal friction because the material is in contact with the channel. In this case, the flow can be rapid or creeping (like viscous deformation), depending on the free-surface gradient. By contrast, in Eq. (18) the potential energy is due only to the vertical drop of the material position $\left(x_{d}-x\right)$ because there is a free-fall of the material that is not in contact with the sliding surface, and the basal and the internal frictions are ineffective. This means that the material does not deform due to the change in the internal pressure or the basal shearing. Therefore, in Eq. (18) the total energy takes the usual form for a rigid body moving in a gravitational field. Moreover, in practice it is desirable to consider kinetic and pressure potential energies, and gravity potential energy together with the friction contribution. Hence, Eq. (15) represents a unified and complete form of the total energy.

\section{Importance of different terms in the total energy function}

It is desirable to properly understand the relative importance of different terms in the total energy function in Eq. (15). The term $m \beta h$ is very important and plays a dominant role when there is a large height gradient. Examples include the situation just after avalanche release and the onset of dambreak flows (both in inclined and horizontal surfaces), and the flow hitting defense structures (Pudasaini and Kröner, 2008). So, $m \beta h$ can cause a massive acceleration or deceleration in either situation. Here, the momentum transfer is mainly due to the hydraulic (pressure) gradient. To correctly model dam-break flows, one must properly take into account the fluid pressure gradient. This arises because in situations of rapidly spreading non-shallow (deep) flows, basal friction $\left(E_{\text {fric }}\right)$ plays virtually no role and the gravity potential $\left(E_{\text {pot }}^{g}\right)$ is dominated by the pressure potential $\left(E_{\text {pot }}^{p}\right)$. On the other hand, for shallow flows, the pressure gradient $(m \beta h)$ is negligible whereas gravity and friction forces are effective. At high elevations, gravity potential plays dominant role, while in the lower part of the channel the friction dominates the energy budget. A part of the gravitational potential energy goes to the kinetic energy ( $\left.E_{\text {kin }}\right)$ while the remaining energy is lost to frictional heat, which goes to the thermal (internal) energy in the granular body, the basal surface and the surrounding. This reduces the momentum of the flow.

\section{Extended Froude number and its significance}

With the definition of the total energy in Eq. (15), we can now define an extended Richardson or Froude number, which accounts for the potential energies, $E_{\mathrm{pot}}^{g}$ and $E_{\mathrm{pot}}^{p}$, associated with the accelerating granular flows down inclines and the pressure. The extended Froude number is defined as the ratio between the kinetic and the potential energy: $F_{r}=u / \sqrt{\beta h+s_{g}\left(x_{d}-x\right)}$. In the classical definition of the shallow-water or granular flow Froude number, the potential energy associated with the surface elevation, $E_{\text {pot }}^{g}=m s_{g}\left(x_{d}-x\right)$, is not considered. The extended Froude number differs substantially from the classical Froude number for higher surface elevations. The term $\beta h$ emerges from the change of the geometry of the moving mass along the slope. Dimensional analysis suggests that for shallow flows $\beta h$ is of order $\ll 1$, while $u$ and $s_{g}\left(x_{d}-x\right)$ are both of order unity. If the mass is shallow with a virtually bed-parallel free-surface, then $\beta h$ can be disregarded. In this situation, if the gravity potential is not taken into account, then Fr is unbounded even though the kinetic energy is bounded. So, formally considering the additional contribution due to the gravitational potential energy, the singularity in Fr is removed. This explains the significance of the new definition of the Froude number. 

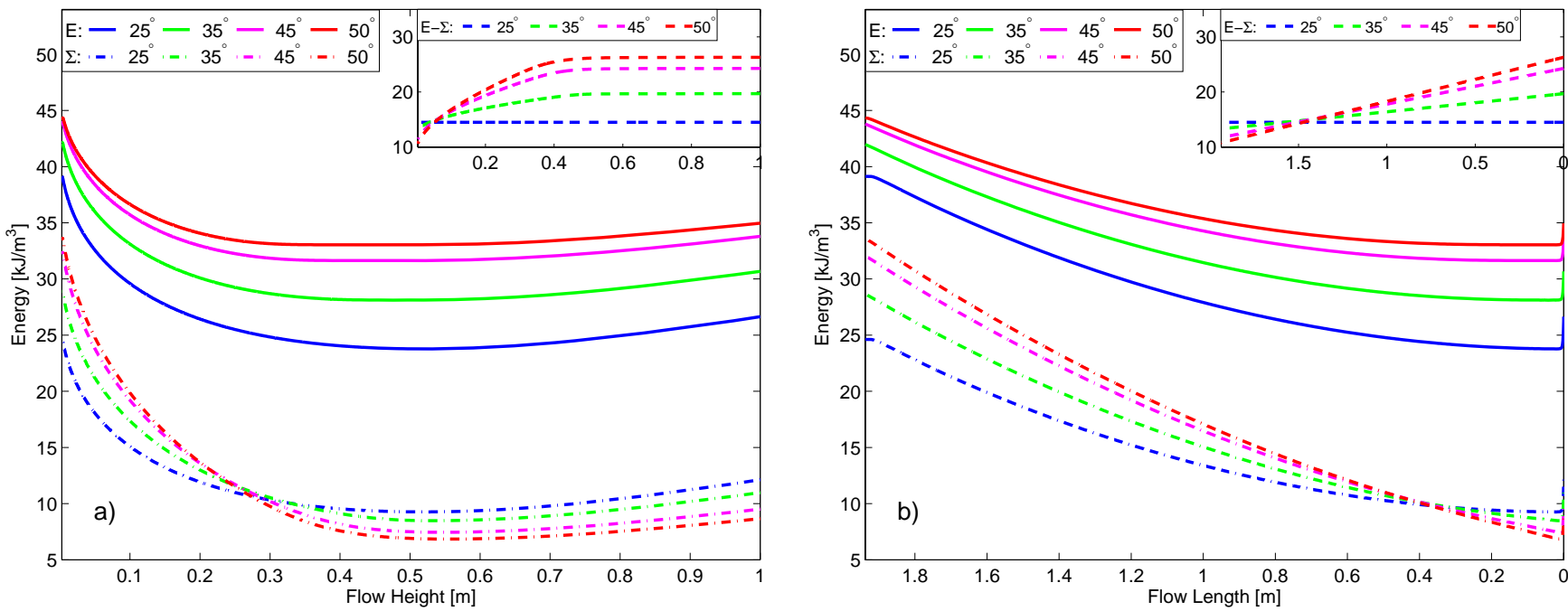

Fig. 1. (a) Energy curves $E, \Sigma$ and difference $E-\Sigma$ in accelerating rapid frictional granular flows down a steep channel with respect to the flow height. (b) Same as in (a) but with respect to the flow length. $u$ and $h$ are obtained from simulation of Eq. (1). Flow is from right to left.

\subsection{Simulation results for the total energy}

In the following we consider the total energy $E$ for a nonstationary flow as given by Eq. (15) and compare it with $\Sigma$, which is defined in Eq. (13) and given by the sum of the kinetic and the potential energy due to pressure. Therefore, $\Sigma$ is the obtained energy if one neglects the net driving acceleration. This comparison allows us to evaluate additional contributions to the total energy of the system due to the potential energy induced by gravity and the frictional heat internal (thermal) energy. We simulated a rapid frictional granular flow down a channel with different inclines. Our analysis is based on uniform material inflow height of $1 \mathrm{~m}$ at the silo gate $(x=0)$, and the outlet velocity is assumed to be $0.5 \mathrm{~ms}^{-1}$. The internal and basal friction angles and the density are set to $\phi=33^{\circ}, \delta=25^{\circ}$, and $\rho=1750 \mathrm{~kg} \mathrm{~m}^{-3}$. The flow variables $u$ and $h$ are obtained by numerically integrating Eq. (1) with TVD-NOC scheme (Pudasaini and Kröner, 2008). It is worth mentioning that the travel distance and the flow height are reciprocally related. This relation depends on many physical and geometrical parameters and flow configurations. As the travel distance increases, the flow height quickly decreases and the flow velocity increases.

In Fig. 1a both energies $E$ and $\Sigma$ are shown as a function of the flow height $h$ for an accelerating flow down a channel with inclines from $\zeta=25^{\circ}$ up to $50^{\circ}$. At the silo gate both potential energies are maximum and the kinetic and the thermal energy are zero. As the flow hits the horizontal reference datum $\left(x=x_{d}\right)$, the gravitational potential energy becomes zero, the pressure potential energy reaches a minimum (or is even negligible), and the kinetic energy and thermal energy attain their maxima. Hence, both $E$ and $\Sigma$ obtain maxima at $x=0$ and $x=x_{d}$. Furthermore, the simulations reveal that as $\zeta$ in- creases, $E$ also increases (for a given $h$ ), because of the associated higher potential energy. But for all slope angles $\Sigma$ is smaller than $E$ because it does not take into account the positive net driving acceleration. Therefore, $\Sigma$ underestimates the associated energy of the flow, as anticipated. This becomes more clear with the energy difference curves, $E-\Sigma$ (in the inset). For the case $\zeta=\delta=25^{\circ}$, the difference is constant over the entire channel (blue line), but for $\zeta>\delta$, the flow is accelerating, $E-\Sigma$ is very large and strongly varies. For a large range of $h$, this difference is bigger for higher differences between slope and basal friction angles, $\zeta-\delta$. This analysis can also be complemented by plotting the energies as a function of the flow length $x$ (Fig. 1b). The total energy decreases very rapidly just below the silo gate because the flow height decreases strongly, as does the pressure potential energy $E_{\mathrm{pot}}^{p}$. This is also the reason for the constant value of the energy difference curve for big flow heights in Fig. 1a. The interesting point in the above analysis is that both total energy functions attain their minima, and that $E$ is always bounded from below by $\Sigma$.

To complete the discussion, we also present the total energy curves for decelerating flows $(\delta>\zeta)$ and the corresponding energy difference curves in Fig. 2. Here, $\phi$ and $\delta$ are kept fixed as before, but the channel slope angles are decreased to $\zeta=25^{\circ}, 20^{\circ}, 15^{\circ}$ and $10^{\circ}$, respectively. Therefore, the flow is driven only by the surface gradient and resisted by the net driving deceleration (basal friction in excess to the gravity load). The results are analogous to Fig. 1, except that the energy difference $(E-\Sigma$, in the inset) is now increasing instead of decreasing along $x$ due to the deceleration. Furthermore, the energy difference is bigger for higher differences $\delta-\zeta$ over a large portion of the channel. So, in general, for an accelerating or decelerating flow the magnitude of $E-\Sigma$ is 

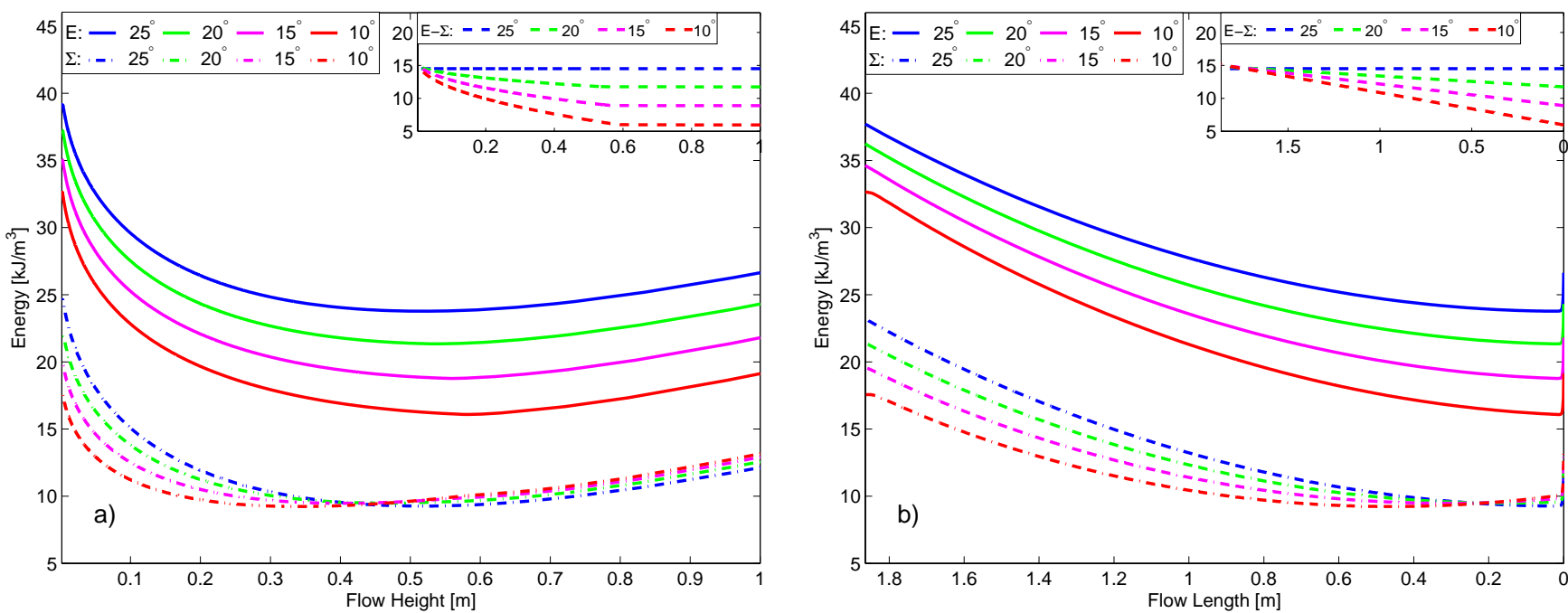

Fig. 2. (a) Energy curves $E, \Sigma$ and difference $E-\Sigma$ in decelerating frictional granular flows down a steep channel with respect to the flow height. (b) Same as in (a) but with respect to the flow length. $u$ and $h$ are obtained from simulation of Eq. (1). Flow is from right to left.

related to the absolute value of $(\zeta-\delta)$. It is very important to properly include the net driving acceleration (deceleration) while calculating the total energy for flows when the friction (gravity) is dominated by gravity (friction). The net driving acceleration (or deceleration) plays an important role in determining the total energy.

\section{Influence of the earth pressure coefficient}

Recently, Pudasaini and Kröner (2008) studied the influence of the earth pressure coefficient on the dynamics of the rapid granular flows down an inclined channel. Their results, as compared with the experimental data, demonstrated that it is essential to employ the anisotropic earth pressure coefficient $(K \neq 1)$ in contrast to the isotropic pressure $(K=1)$. For extensional flow, with $\phi=33^{\circ}, \delta=25^{\circ}, K=0.7656$, which is less than unity. Therefore, by using $K=1$, the pressure potential energy would be increased non-physically by more than $23 \%$. This influence is substantial when the flow height and the difference in the friction angles $(\phi-\delta)$ are larger, mainly in the vicinity of the silo gate. The influence of $K$ is larger for $\Sigma$ than for $E$.

\section{Total energy of the experimental granular channel flow}

We now consider an experiment with granular quartz particles of mean diameter $5 \mathrm{~mm}$ flowing down a $2 \mathrm{~m}$ long steep rectangular channel inclined at an angle $50^{\circ}$. The opening gap of the silo gate is $0.06 \mathrm{~m}$ and $\phi$ and $\delta$ are as before (Pudasaini et al., 2007). The total energy curves, both with respect to the flow height and the flow length, are shown in Fig. 3. The data is used to analyze the lower part of the channel. As explained before, the energy curves show large deviations of
$\Sigma$ from $E$. However, we do not have enough data to show how these energy curves would attain their minima like in Fig. 1. As inferred from the simulation, for $\Sigma$ the energy minimum lies on the right side of the curve whilst for $E$ on the left. Moreover, Figs. 1 and 3 represent similar qualitative behavior but for different boundary conditions.

The curves $E-\Sigma$ in Figs. 1-3 clearly demonstrate that the potential energy $E_{\text {pot }}^{g}$ and the friction energy $E_{\text {fric }}$ can not be neglected in the energy considerations for an accelerating (or decelerating) flow of frictional granular materials down sloping surfaces. Otherwise, one gets a substantial discrepancy to the actual total energy. This discrepancy can be of the order of tens of kilojoule per cubic meter of material for even one meter or a few centimeters of silo gate opening. This would be substantially higher for natural avalanches or debris flows consisting of up to $10^{12} \mathrm{~m}^{3}$ of material.

\section{Conclusions}

This paper considered the total energy $E$; carried out by the rapidly deforming and flowing frictional granular material down inclined slope; that takes into account the net driving acceleration of the system, spatio-temporal variations in flow height and the flow velocity. To derive an expression for $E$, we introduced an auxiliary energy function $\mathcal{E}$ which manifests itself as a solitary wave. $\mathcal{E}$ has some interesting features. It is related to the determinant of the corresponding matrix of the flow equations. The spatial derivative of $\mathcal{E}$ is the net-acceleration of the system and thus it is a constant if the system does not accelerate. For the case of an accelerating but stationary flow, this function is given by the sum of the kinetic and pressure potential energy associated with 

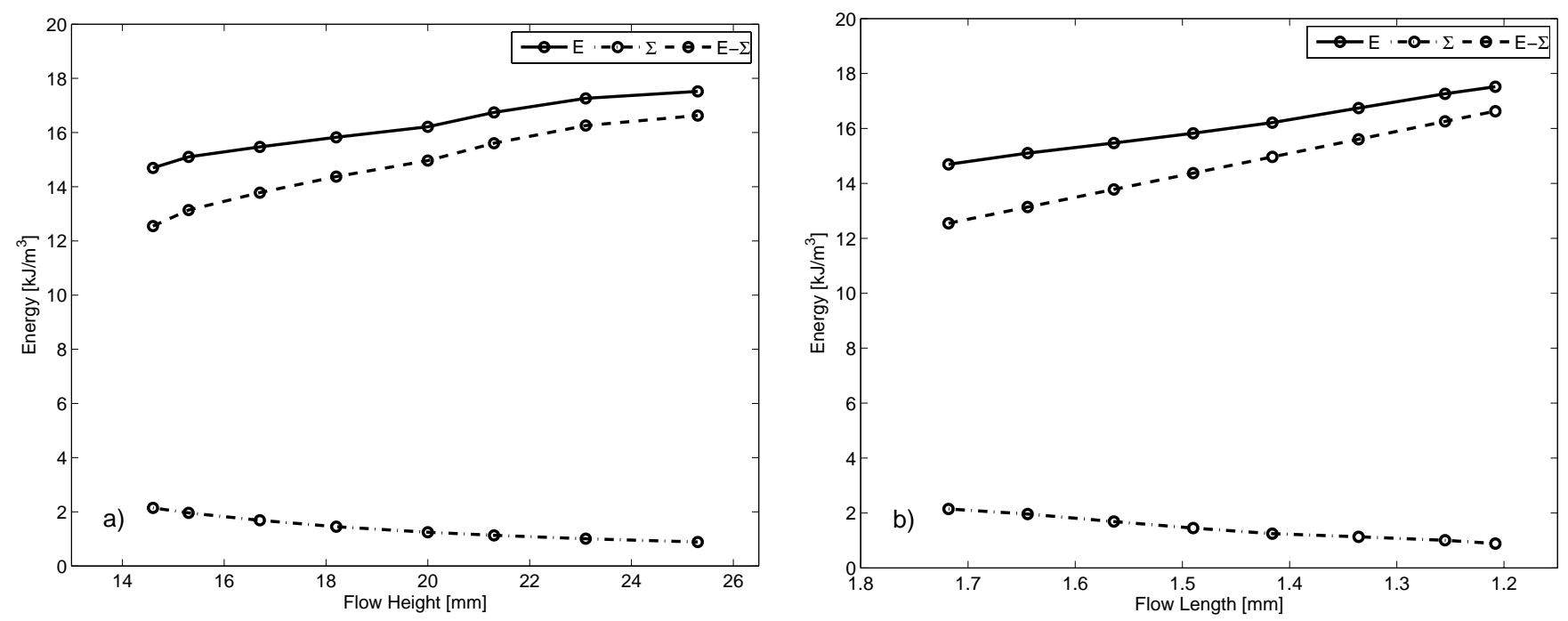

Fig. 3. (a) Energy curves $E, \Sigma$ and difference $E-\Sigma$ in an accelerating rapid frictional granular flow down a steep channel with respect to the flow height. (b) Same as in (a) but with respect to the flow length. $u$ and $h$ are obtained from the experimental data (Pudasaini et al., 2007). Flow is from right to left.

the variations in flow height and bulk deformations. Therefore, $\mathcal{E}$ supplies these two energy contributions to the total energy $E$, which additionally takes into account the gravitational potential and the dissipative frictional energy essential for modelling granular flows. The dissipative frictional energy is induced by the Coulomb friction force acting at the basal surface. It characterizes, together with the pressure potential energy (which takes into account the extensional or compressional nature of the flow with an anisotropic earth pressure coefficient), the whole frictional behavior of the granular flow. Since the force induced by the free-surface gradient is not conservative, the total energy $E$ is not constant for non-stationary flows. It is a conserved quantity only for a stationary flow. If the flow is non-accelerating the total mechanical energy of the flow, as the sum of the kinetic and the potential energy due to pressure, always remains constant. When the media is an ideal fluid and the net-driving force vanishes, the total energy reduces to an often used energy form. Simulation and experimental results demonstrate that the influence of the net-driving force and the geometric deformation of the sliding mass on the total energy $E$ is substantial. We have also defined the extended Froude number that differs substantially from the classical thin-film flow Froude number for higher surface elevations in that it takes into account the additional contribution due to gravitational potential energy not previously considered. Finally, we mention that this paper enhances our understanding of mass flows and offers an explicit expression for the total energy for accelerating flows of frictional material down inclined slopes. Practitioners can find the energy functions derived here useful in dealing with the avalanche defense, hazard mapping and planning. A practical application is to help derive engineering formulas describing the dissipation of flow energy when avalanches strike retarding structures such as catching dams and breaking mounds.

Acknowledgements. We thank the German Research Foundation (DFG) for the financial support through the project PU 386/1-1: Transition of a granular flow into the deposit. We are grateful to S. A. Miller, K. Hutter, S. Noelle and A. Zoporowski for fruitful discussions. Special thanks are also due to J. McElwaine, P. Bartelt, E. Pelinovsky and two other anonymous reviewers for their constructive reviews that helped to improve the quality of the paper.

Edited by: R. Grimshaw

Reviewed by: J. McElwaine, P. Bartelt, E. Pelinovsky, and 2 other anonymous Referees

\section{References}

Bartelt, P., Buser, O., and Kern, M.: Dissipated work, stability and the internal flow structure of granular snow avalanches, J. Glaciol., 51(172), 125-138, 2005.

Bartelt, P., Buser, O., and Platzer, K.: Fluctuation-dissipation relations for granular snow avalanches, J. Glaciol., 52(179), 631643, 2006.

Bartelt, P., Buser, O., and Platzer, K.: Starving avalanches: Frictional mechanisms at the tails of finite-sized mass movements, Geophys. Res. Lett., 34, L20407, doi:10.1029/2007GL031352, 2007.

Bouchut, F., Mangeney-Castelnau, A., Perthame, B., and Vilotte, J.-P.: A new model of Saint Venant and Savage-Hutter type for gravity driven shallow water flows, C. R. Math, 336(6), 531-536, doi:10.1016/S1631-073X(03)00117-1, 2003. 
Buser, O. and Bartelt, P.: Production and decay of random kinetic energy in granular snow avalanches, J. Glaciol., 55(189), 3-12, 2009.

Castro, M., Gallardo, J. M., and Parés, C.: High order finite volume schemes based on reconstruction of states for solving hyperbolic systems with nonconservative products. Applications to shallowwater systems, Math. Comput., 75(255), 1103-1134, 2006.

Dutykh, D. and Dias, F.: Energy of tsunami waves generated by bottom motion, P. R. Soc. A, 465, 725-744, 2009.

Erismann, T. H. and Abele, G.: Dynamics of Rockslides and Rockfalls, Springer, Berlin, Germany, 2001.

Fine, I. V., Rabinovich, A. B., Thomson, R. E., and Kulikov, E. A.: Numerical modeling of tsunami generation by submarine and subaerial landslides, in: Submarine Landslides and Tsunamis, edited by: Yalciner, A. C., Pelinovsky, E., Okal, E., and Synolakis, C. E., Kluwer Academic Publishers, Netherlands, 69-88, 2003.

Gray, J. M. N. T., Wieland, M., and Hutter, K.: Gravity-driven free surface flow of granular avalanches over complex basal topography, P. R. Soc. A, 455, 1841-1874, 1999.

Gwiazda, P.: On measure-valued solutions to a two-dimensional gravity-driven avalanche flow model, Math. Method Appl. Sci., 28, 2201-2223, 2005.

Heim, A.: Bergsturz und Menschenleben, Fretz \& Wasmuth, Zürich, 1932.

Hsü, K.: On sturzstroms-catastrophic debris streams generated by rockfalls, Geol. Soc. Am. Bull., 86, 129-140, 1975.

Jin, S. and Wen, X.: An efficient method for computing hyperbolic systems with geometrical source terms having concentrations, J. Comput. Math., 22, 230-249, 2004.

Le Roux, A. Y.: Riemann solvers for some hyperbolic problems with a source term, in: ESIAM Proceedings/Actes du 30EME Congres d'Analyse Numerique: CANum'98, 75-90, 1998.

Mangeney, A., Heinrich, P., and Roche, R.: Analytical solution for testing debris avalanche numerical models, Pure Appl. Geophys., 157, 1081-1096, 2000.

Noelle, S., Pankratz, N., Puppo, G., and Natvig, J.: Well-balanced finite volume schemes of arbitrary order of accuracy for shallow water flows, J. Comput. Phys., 213, 474-499, 2006.

Noelle, S., Xing, Y., and Shu, C.-W.: High Order Well-balanced Finite Volume WENO Schemes for Shallow Water Equation with Moving Water, J. Comput. Phys., 226, 29-58, 2007.

Pudasaini, S. P. , Hsiau, S.-S., Wang, Y., and Hutter, K.: Velocity measurements in dry granular avalanches using Particle Image Velocimetry-Technique and comparison with theoretical predictions, Phys. Fluids, 17(9), 93301, doi:10.1063/1.2007487, 2005.
Pudasaini, S. P. and Hutter, K.: Rapid Shear Flows of Dry Granular Masses Down Curved and Twisted Channels, J. Fluid Mech., 495, 193-208, 2003.

Pudasaini, S. P. and Hutter, K.: Avalanche Dynamics: Dynamics of Rapid Flows of Dense Granular Avalanches, Springer, Berlin, Germany, 2007.

Pudasaini, S. P., Hutter, K., Hsiau, S.-S., Tai, S.-C., Wang, Y., and Katzenbach, R.: Rapid Flow of Dry Granular Materials down Inclined Chutes Impinging on Rigid Walls, Phys. Fluids, 19(5), 053302, doi:10.1063/1.2726885, 2007.

Pudasaini, S. P. and Kröner, C.: Shock waves in rapid flows of dense granular materials: Theoretical predictions and experimental results, Phys. Rev. E, 78(4), 041308, doi:10.1103/PhysRevE.78.041308, 2008.

Pudasaini, S. P., Wang, Y., and Hutter, K.: Modelling Debris Flows Down General Channels, Nat. Hazard Earth Sys., 5, 799-819, 2005.

Pudasaini, S. P., Wang, Y., and Hutter, K.: Rapid motions of freesurface avalanches down curved and twisted channels and their numerical simulation, Philos. T. R. Soc. A, 363(1832), 15511571, 2005.

Pudasaini, S. P. , Wang, Y., Sheng, L.-T., Hsiau, S.-S., Hutter, K., and Katzenbach, R.: Avalanching granular flows down curved and twisted channels: Theoretical and experimental results, Phys. Fluids, 20(7), 073302, doi:10.1063/1.2945304, 2008.

Rao, N. M.: Avalanche Protection and Control in the Himalayas, Defence. Sci. J., 35(2), 255-266, 1985.

Rudenko, O. V., Sobisevich, A. L., and Sobisevich, L. E.: Nonlinear dynamics of slope flows: simple models and exact solutions, Dokl. Earth Sci. 416(7), 1109-1113, 2007.

Saint-Venant, A. J. C.: Theorie du mouvement non-permanent des eaux, avec application aux crues des rivieres et a l'introduction des marees dans leur lit, Comptes rendus des seances de l'Academie des Sciences, 36, 174-154, 1871.

Savage, S. B. and Hutter, K.: The motion of a finite mass of granular material down a rough incline, J. Fluid Mech., 199, 177-215, 1989.

Ui, T.: Volcanic dry avalanche deposits-identification and comparison with nonvolcanic debris stream deposits, J. Volcanol. Geoth. Res., 18, 135-150, doi:10.1016/0377-0273(83)90006-9, 1983.

Ward, S. N. and Day, S.: Particulate kinematic simulations of debris avalanches: interpretation of deposits and landslide seismic signals of Mount Saint Helens, 1980 May 18., Geophys. J. Int., 167, 991-1004, 2006. 